\title{
Glass transition memorized by the enthalpy- entropy compensation in the shear thinning of supercooled metallic liquids
}

\author{
Meng Zhang ${ }^{1,2,3}$ (b) and Lin Liu ${ }^{3}$ \\ ${ }^{1}$ Institute of Advanced Wear and Corrosion Resistant and Functional Materials, Jinan University, \\ Guangzhou 510632, People's Republic of China \\ 2 State Key Laboratory of Nonlinear Mechanics, Institute of Mechanics, Chinese Academy of Sciences, \\ Beijing 100190, People's Republic of China \\ ${ }^{3}$ School of Materials Science and Engineering, Huazhong University of Science and Technology, Wuhan \\ 430074, People's Republic of China \\ E-mail: m.zhangiwrm@jnu.edu.cn
}

Received 29 March 2018, revised 27 April 2018

Accepted for publication 3 May 2018

Published 21 May 2018

\begin{abstract}
To unravel the true nature of glass transition, broader insights into glass forming have been gained by examining the stress-driven glassy systems, where strong shear thinning, i.e. a reduced viscosity under increasing shear rate, is encountered. It is argued that arbitrarily small stress-driven shear rates would 'melt' the glass and erase any memory of its thermal history. In this work, we report a glass transition memorized by the enthalpy-entropy compensation in strongly shear-thinned supercooled metallic liquids, which coincides with the thermal glass transition in both the transition temperature and the activation Gibbs free energy. Our findings provide distinctive insights into both glass forming and shear thinning, and enrich current knowledge on the ubiquitous enthalpy-entropy compensation empirical law in condensed matter physics.
\end{abstract}

Keywords: metallic glasses, glass transition, shear thinning, enthalpy-entropy compensation

S] Supplementary material for this article is available online

(Some figures may appear in colour only in the online journal)

\section{Introduction}

The effort to understand the mysterious glass formation phenomenon [1] is frustratingly hindered by the procedure known as aging [2,3], in which glasses spontaneously evolve extremely slowly towards the equilibrium state due to their out-of-equilibrium nature. To circumvent this situation, stressdriven glassy systems which exhibit strong shear thinning (i.e. a reduced viscosity under increasing shear rate) have been intensively examined [4-10]. The stress-driven flow characterized by a shear rate will create a non-equilibrium while steady state, in which the aging of glassy systems is interrupted and the time translation invariance is recovered $[5,11]$. This steady state can thus be examined to provide broader insights into glassy physics, by easily adopting the shear rate rather than the natural aging time of glasses as the control parameter. As evidenced by the effective temperature $[5,12]$ and the most probable atomic displacement [13], a scaling relationship between stress and temperature as they affect the glassy systems has been confirmed as both of them could reduce the viscosity and 'melt' a glass into a liquid [14-16]. Hence, it is argued that an arbitrarily small shear rate exerted on a glass is able to melt the glass into a liquid and thus erase any memory of its thermal history in glassy state $[5,6,11]$.

However, in this work, by virtue of the thermodynamic enthalpy-entropy compensation law (or known as the MeyerNeldel (MN) rule) [17] which reads $\Delta H=T_{\mathrm{MN}} \Delta S$ and prevails in diverse processes [18-21] in condensed matter physics, where $\Delta H$ is the activation enthalpy; $\Delta S$ is the activation entropy; and $T_{\mathrm{MN}}$ is the iso-kinetic compensation temperature, 
we found a glass transition memorized in strongly shearthinned supercooled liquids. The memorized glass transition coincides with the thermal glass transition in both the glass transition temperature and the activation Gibbs free energy $\Delta G$, suggesting a kinetic nature of the glass forming process and an intrinsic correlation between thermal glass forming and stress-driven shear thinning. The origin of the enthalpyentropy compensation in supercooled liquids is attributed to the multiple-excitation effect [22], i.e. the facilitation effect and the correlation effect in the activation of shear transformation zones (STZs) [23-25].

\section{Methods and experimental}

Due to the structural similarity to the dense random packing of spheres [26], supercooled metallic liquids (SMLs) represent an ideal research subject for the current study. The enthalpy-entropy compensation law in the shear thinning of $\mathrm{Zr}_{58.5} \mathrm{Cu}_{15.6} \mathrm{Al}_{10.3} \mathrm{Ni}_{12.8} \mathrm{Nb}_{2.8}$ (Vit106a) and $\mathrm{Zr}_{41.2} \mathrm{Ti}_{13.8} \mathrm{Cu}_{12.5} \mathrm{Ni}_{10} \mathrm{Be}_{22.5}$ (Vit1) SMLs are verified by the linear relationship between the activation entropy $\Delta S$ and the activation enthalpy $\Delta H$ in the flow of SMLs.

To determine the activation entropy $\Delta S$ and the activation enthalpy $\Delta H$, the transition state theory (TST) based flow model of SMLs [27, 28] is adopted, where STZ is envisioned as the elementary excitation event of the thermally assisted activation process in the flow of SMLs. In the STZ model, the flow equation of SMLs reads [27]: $\dot{\varepsilon}=\dot{\varepsilon}_{0} \exp \left(-\Delta G(T, \sigma) / k_{\mathrm{B}} T\right)$, where $\Delta G(T, \sigma)=\Delta H-T \Delta S=\Delta Q-\sigma V-T \Delta S$ is the activation Gibbs free energy for the flow of SML. $\dot{\varepsilon}$ is the strain rate. $\dot{\varepsilon}_{0}$ is a pre-factor that defines the upper limit of the flow rate of SMLs. $\sigma$ is the steady state flow stress. $T$ is temperature. $k_{\mathrm{B}}$ is Boltzmann's constant. $\Delta Q$ is the activation energy for the flow of SML. $V$ is the activation volume for the flow of SML. Since the temperature dependence of $\Delta S$ and $\Delta H$ is weak [19], $\Delta S$ and $\Delta H$ are considered as constants at given flow stress. Therefore, based on the linear relationship: $\Delta G=\Delta H-T \Delta S$, the crucial step to derive $\Delta S$ and $\Delta H$ is to determine the dependence of $\Delta G$ on temperature $T$. Whereby, $\Delta S$ can be determined from the slope of the linear relationship ( $\Delta G$ versus $T$ ), and $\Delta H$ can be determined from the intercept of the linear relationship ( $\Delta G$ versus $T$ ) with the coordinate axis. It is noted that $\Delta G(T, \sigma)$ is a function of both temperature $T$ and flow stress $\sigma$. Under different flow stresses $\sigma$, different linear relationships ( $\Delta G$ versus $T$ ) can be derived. Therefore, the relationship between the activation entropy $\Delta S$ and the activation enthalpy $\Delta H$ at different flow stresses in the flow of SMLs can be examined.

As a function of temperature $T$ and flow stress $\sigma$, $\Delta G(T, \sigma)$ can be written as $\Delta G(T, \sigma)=k_{\mathrm{B}} T \ln \left(\dot{\varepsilon}_{0} / \dot{\varepsilon}\right)$ based on the above flow equation. In Argon's seminal work [29], $\dot{\varepsilon}_{0}$ reads $\dot{\varepsilon}_{0}=\alpha v_{0} \varepsilon_{\text {unit }}$, where $\alpha$ is a geometric factor depending on the shape of STZ; $v_{0}$ is the attempt frequency; and $\varepsilon_{\text {unit }}$ is the unit strain carried by an STZ. Specifically, $\alpha$ is in the range $0.1-0.2$. $v_{0}$ is of a order of magnitude of $10^{12} \mathrm{~Hz}$. $\varepsilon_{\text {unit }}$ is also of a order of 0.1 . Therefore, to determine the temperature and flow stress together dependent $\Delta G(T, \sigma)$, the steady state flow stress $\sigma$ of the 2 SMLs are measured in a wide range of strain rate $\dot{\varepsilon}$ from $10^{-3} \mathrm{~s}^{-1}$ to $10^{0} \mathrm{~s}^{-1}$ and at different temperatures $T$ (see supplemental materials SI (stacks. iop.org/JPhysCM/30/245401/mmedia) for more details on the experimental tests, figures $\mathrm{S} 1$ and $\mathrm{S} 2$ ), as shown in figures 1(a) and (b). Based on the combination $(T, \sigma(\dot{\varepsilon}))$ of the experimental temperature $T$ and the measured steady state flow stress at different strain rates $\sigma(\dot{\varepsilon})$, the dependence of $\Delta G$ on temperature and flow stress of the 2 SMLs can be obtained by substituting the corresponding strain rate into the equation: $\Delta G(T, \sigma)=k_{\mathrm{B}} T \ln \left(\dot{\varepsilon}_{0} / \dot{\varepsilon}\right)$. Consequently, the activation entropy $\Delta S$ and activation enthalpy $\Delta H$ in the flow of SMLs under different flow stresses can be readily derived by fitting the dependence of $\Delta G$ on temperature with the linear relationship: $\Delta G=\Delta H-T \Delta S$, as will be shown in the following sections.

\section{Results and discussion}

\subsection{Activation enthalpy and activation entropy}

Figures 1(c) and (d) show the strain rate sensitivity index $m^{*}=\partial \ln \sigma / \partial \ln \dot{\varepsilon}$ derived from the steady state flow stress $\sigma$ at different strain rates $\dot{\varepsilon}$ of the 2 SMLs (see supplemental materials SI, figure S2). A clear decrease of $m^{*}$ from $\sim 0.7$ to nearly 0 is displayed, indicating a strong shear thinning phenomenon in the steady state flow of the 2 SMLs at different temperatures. Figures 2(a) and (b) plot the $\Delta G$ derived from $\Delta G(T, \sigma)=k_{\mathrm{B}} T \ln \left(\dot{\varepsilon} / \dot{\varepsilon}_{0}\right)$ against the steady state flow stress $\sigma$ of Vit106a and Vit1 SMLs at different temperatures $T$, respectively. It can be seen that $\Delta G$ decreases monotonously with both increasing $\sigma$ and increasing $T$. This phenomenon reflects the stress-temperature scaling in the activation dynamics of STZs in the steady state flow of SMLs [14, 30]. This is because that the effect of $\sigma$ similar to $T$ could also reduce the activation energy barrier on the local potential energy landscape, thereby 'melting' a glass into a liquid. With the group of $\Delta G$ versus $\sigma$ relations at different temperature $T$ presented in figures 2(a) and (b), the $\Delta G$ versus $T$ relations under different flow stresses can be conveniently interpolated.

Figures 3(a) and (b) show the $\Delta G$ versus $T$ relations interpolated from figure 2 to the first approximation for Vit106a and Vit1 SMLs, respectively. It can be seen that, consistent with figure $2, \Delta G$ decreases monotonously with increasing $T$ at constant flow stress and decreases with increasing $\sigma$ at constant temperature. Linear fits of the relationship between $\Delta G$ and $T$ can be obtained for the 2 SMLs, except for small deviations of $\Delta G$ at $633 \mathrm{~K}$ for Vit1 SML, which will be discussed later. The linear relationship between $\Delta G$ and $T$ confirms that $\Delta S$ and $\Delta H$ are approximately independent of temperature and could be treated as constants at given flow stress. Based on the equation: $\Delta G=\Delta H-T \Delta S, \Delta S$ is determined from the slope of the fit of $\Delta G$ versus $T$ relations; $\Delta H$ is determined from the intercept of the fit with the longitude axis; and $T_{\Delta G 0}$ where $\Delta G$ equals $0 \mathrm{KJ} \mathrm{mol}^{-1}$ is determined from the intercept of the fit with the transversal axis.

It is important to note an increasing slope of the fit of the $\Delta G$ versus $T$ relations, i.e. an increasing $\Delta S$, under 

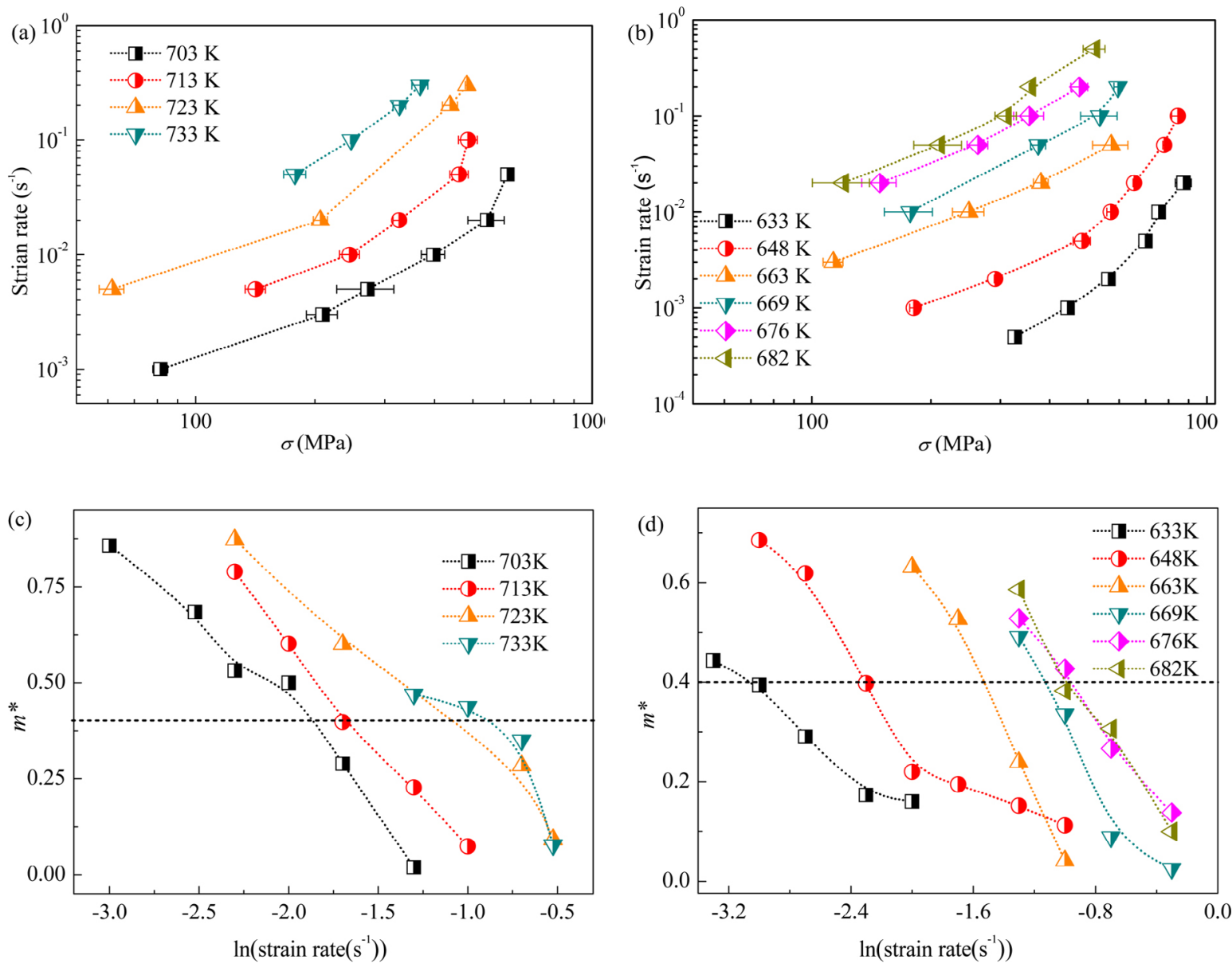

Figure 1. The steady state flow stress of SMLs: (a) Vit106a, (b) Vit1; and the strain rate sensitivity index $m^{*}$ in the steady flow of SMLs: (c) Vit106a, (d) Vit1. The dotted lines are eye guides.

increasing flow stress for the 2 SMLs. For clarity, figure 3(c) shows the evolution of $\Delta S$ under increasing flow stress for Vit106a and Vit1 SMLs. It can be seen that $\Delta S$ increases with increasing flow stress, though different increasing tendencies are exhibited. According to the TST, $\Delta S$ is the difference of entropy between the initial state and the activated state. Thus, figure 3(c) demonstrates that higher flow stresses lead to more disordered structures, i.e. structural disordering, because larger entropy means more disorder. The structural disordering arises from the phenomenon that the activation of STZs in the flow of SMLs would lead to an increment in the concentration of free volume [29]. A higher free volume concentration indicates a more disordered structure. The observation in figure 3(c) agrees well with the structural disordering observed in the flow of SMLs in previous experimental tests where the increment of free volume concentration is detected $[31,32]$. It is also noted that the approximate saturation of $\Delta S$ for Vit1 SML at high stress levels is probably due to the extreme of structural ordering is reached, as have been reported in the literatures [31, 32]. Different increasing tendency of $\Delta S$ would indicate different rheology dynamics of different SMLs. More details on the picture of the development of structural disordering in the
STZ-mediated flow of SMLs [33, 34] can be found in the seminal work of Argon's [29].

Due to the increasing $\Delta S$ under increasing flow stress, the $\Delta G$ versus $T$ relations diverge from each other towards higher $T$, as shown in figures 3(a) and (b). In turn, as illustrated in figure $3\left(\mathrm{~d}\right.$ ), a group of $T_{\Delta G 0}$ (from $948 \mathrm{~K}$ to $1161 \mathrm{~K}$ for Vit106a, from 940 to $1078 \mathrm{~K}$ for Vit1), rather than a constant value [35], can be observed. It is noted that the liquidus temperature of Vit106a metallic glass is $1110 \mathrm{~K}$ [36] and the liquidus temperature of Vit1 metallic glass is $993 \mathrm{~K}$ [37], coinciding with the range of $T_{\Delta G 0}$. As $T_{\Delta G 0}$ represents the temperature where $\Delta G$ for flow equals $0 \mathrm{KJ} \mathrm{mol}^{-1}$, it is rational that the 2 groups of $T_{\Delta G 0}$ of the 2 SMLs coincide with their liquidus temperature. The group of $T_{\Delta G 0}$ can be rationalized with the observation of an underlying polyamorphic transition in the yield of glassy polymers [38] where different flow stresses would incubate different amorphous states which reasonably would lead to different $T_{\Delta G 0}$. It is also noted that the compensation effect between $\Delta S$ and $\Delta H$ in the plastic deformation of metallic glasses has been reported recently [27]. However, an increasing $\Delta S$ was not observed, probably due to the different regimes of deformation examined as will be discussed later. 


\subsection{Glass transition memorized by the enthalpy-entropy compensation}

Figures 4(a) and (b) show the linear relationship between $\Delta H$ and $\Delta S$ of Vit106a and Vit1 SMLs, respectively. It can be seen that the compensation law between $\Delta H$ and $\Delta S$ is satisfied as $\mathrm{d} \Delta H / \mathrm{d} \Delta S=T_{\mathrm{MN}}$. This linear relationship between $\Delta H$ and $\Delta S$ is virtually determined by the linear relationship between $\Delta G$ and $T$. The slope of the linear fit of $\Delta G$ versus $T$ relation is $\Delta S$. The intercept of the linear fit with the longitude axis is $\Delta H$. Plotted against $\Delta S$, the group of $\Delta H$ would exactly collapse onto a line as shown in figures 4 (a) and (b), on condition that all the fits of the $\Delta G$ versus $T$ relations intersect with each other on the same critical point (see supplemental materials SII). As illustrated in figure 3(d), due to the increasing $\Delta S$ under increasing flow stress in shear thinning, the $\Delta G$ versus $T$ relations converge towards lower temperature and memorizes a critical temperature $T_{\mathrm{MN}} \sim 0.96 T_{\mathrm{g}}(657 \mathrm{~K}$ and $597 \mathrm{~K}$ for Vit106a and Vit1, for which $T_{\mathrm{g}}$ are $683 \mathrm{~K}$ and $625 \mathrm{~K}$, respectively), which can be derived from the slope of $\Delta H$ versus $\Delta S$ relation.

On the $\Delta G$ versus $T$ diagram, this critical point indicates that, as $T$ decreases towards $T_{\mathrm{MN}}, \Delta G$ under different flow stresses increase gradually and become identical as $\sim 178 \mathrm{KJ} \mathrm{mol}^{-1}$ for Vit1 and as $\sim 183 \mathrm{KJ} \mathrm{mol}^{-1}$ for Vit106a (approximately the activation energy of glass transition $\Delta G_{\mathrm{g}} \approx 220-250 \mathrm{KJ} \mathrm{mol}^{-1}$ for the 2 SMLs, see supplemental materials SII. Figure S5). Namely, as predicted by the TST, in the steady state flow of SMLs approaching the critical temperature $T_{\mathrm{MN}}$, the strain rates under different flow stresses gradually slow down and approximate a finite small constant value $\dot{\varepsilon}_{\mathrm{MN}}=\dot{\varepsilon}_{0} \exp \left(-\Delta G_{\mathrm{MN}} / k_{\mathrm{B}} T_{\mathrm{MN}}\right) \approx 10^{-6} \mathrm{~s}^{-1}$. More importantly, as illustrated in figure $3(\mathrm{~d})$, it is noted that, as the temperature decreases towards $T_{\mathrm{MN}}, \Delta G$ under a higher flow stress $\left(\Delta G_{\sigma 1}\right.$ in figure 3(d)) increases faster than $\Delta G$ under a lower flow stresses ( $\Delta G_{\sigma 6}$ in figure 3(d)), because of the larger activation entropy $\Delta S$ under higher flow stresses as shown in figure $3(\mathrm{c})$. Based on the TST, it means that the flow of SML under higher flow stresses slows down faster, i.e. faster dynamics undergoes faster 'frozen', as the temperature decreases towards $T_{\mathrm{MN}}$. More precisely, at the critical point $T_{\mathrm{MN}}$, the flow rate of SML cannot be further increased above $10^{-6} \mathrm{~s}^{-1}$ by increasing the flow stress, indicating a diverging resistance to accelerated flow. Namely, as the temperature decreases towards $T_{\mathrm{MN}}$, the enthalpy-entropy compensation requires that the SMLs under different flow stresses should be kinetically 'frozen' into an identical solid-like steady flow state [2]. The limit case is that the constant strain rate $\dot{\varepsilon}_{\mathrm{MN}}$ equals $0 \mathrm{~s}^{-1}$ at $T_{\mathrm{MN}}$ and the SML becomes a solid, i.e. a glass. It is also noted that a strain rate of $10^{-6} \mathrm{~s}^{-1}$ under a steady flow stress of $10^{2} \mathrm{MPa}$ corresponds to a viscosity of $10^{14} \mathrm{~Pa} \cdot \mathrm{s}$, which is the typical viscosity value at which the SML is assumed to transit into a glass [2]. Hence this critical point $\left(T_{\mathrm{MN}}, \Delta G_{\mathrm{MN}}\right)$ on the $\Delta G$ versus $T$ diagram is a clear reminiscent of the thermal glass transition. Considering the ambiguity in the measurement of $T_{\mathrm{g}}$ [39] and the almost consistent activation Gibbs free energy $\Delta G_{\mathrm{MN}} \approx \Delta G_{\mathrm{g}}$, the isokinetic temperature $T_{\mathrm{MN}} \sim 0.96 T_{\mathrm{g}}$ memorized by the
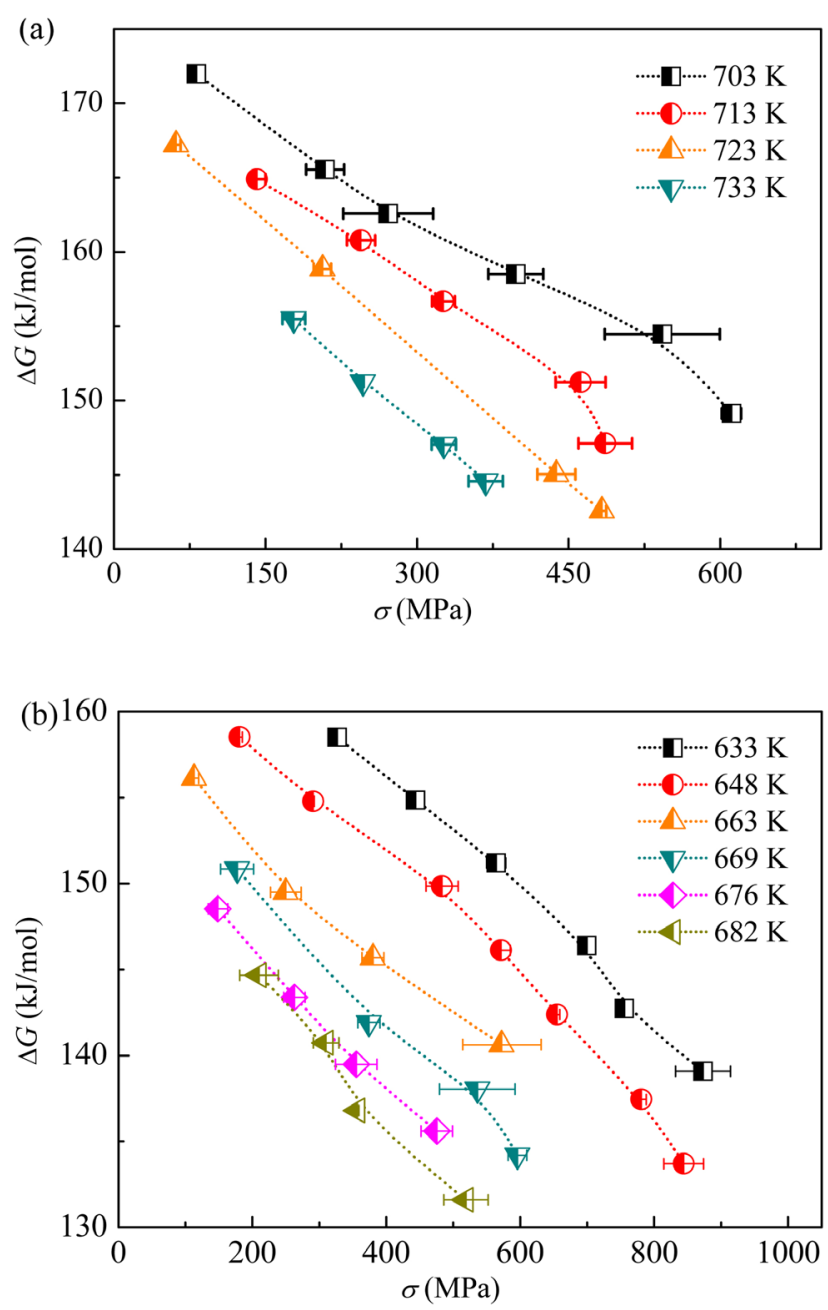

Figure 2. The activation Gibbs free energy versus flow stress ( $\Delta G$ versus $\sigma$ ) relations in the steady flow of SMLs at different temperatures: (a) Vit106a, (b) Vit1. The dotted lines are eye guides.

$\Delta H-\Delta S$ compensation is concluded to be another version of the glass transition temperature $T_{\mathrm{g}}$. This memorized glass transition suggests a kinetic nature of the glass forming process. It is of great importance to note that this transition of SMLs into glasses as the temperature approaching $T_{\mathrm{MN}}$ (i.e. the SML deviates from its meta-equilibrium state) is virtually memorized by the intrinsic shear disordering of SMLs (i.e. an increasing activation entropy). The memorized glass transition thus suggests an intrinsic correlation between glass forming and shear thinning. The values of $T_{\mathrm{MN}}$ at $633 \mathrm{~K}$ for Vit1 SML(close to the measured $T_{\mathrm{g}}=625 \mathrm{~K}$ ) in figure $3(\mathrm{~b}$ ) deviating from the linear fit could also be rationalized by the deviation of the Vit1 SML from its meta-equilibrium state as the temperature decrease towards $T_{\mathrm{MN}}$, because closer to the glass transition, the homogeneous steady flow state is getting more difficult to reach in real experimental tests [40]. Moreover, this deviation of $\Delta G$ close to $T_{\mathrm{g}}$ also interprets the fact that the current flow data of the 2 SMLs fails to examine the compensation effect in the deformation of metallic glasses in glassy state [27].

More intriguingly, as illustrated in figure $3(\mathrm{~d})$, in the regime where $T<T_{\mathrm{MN}}$, a higher flow stress would result 

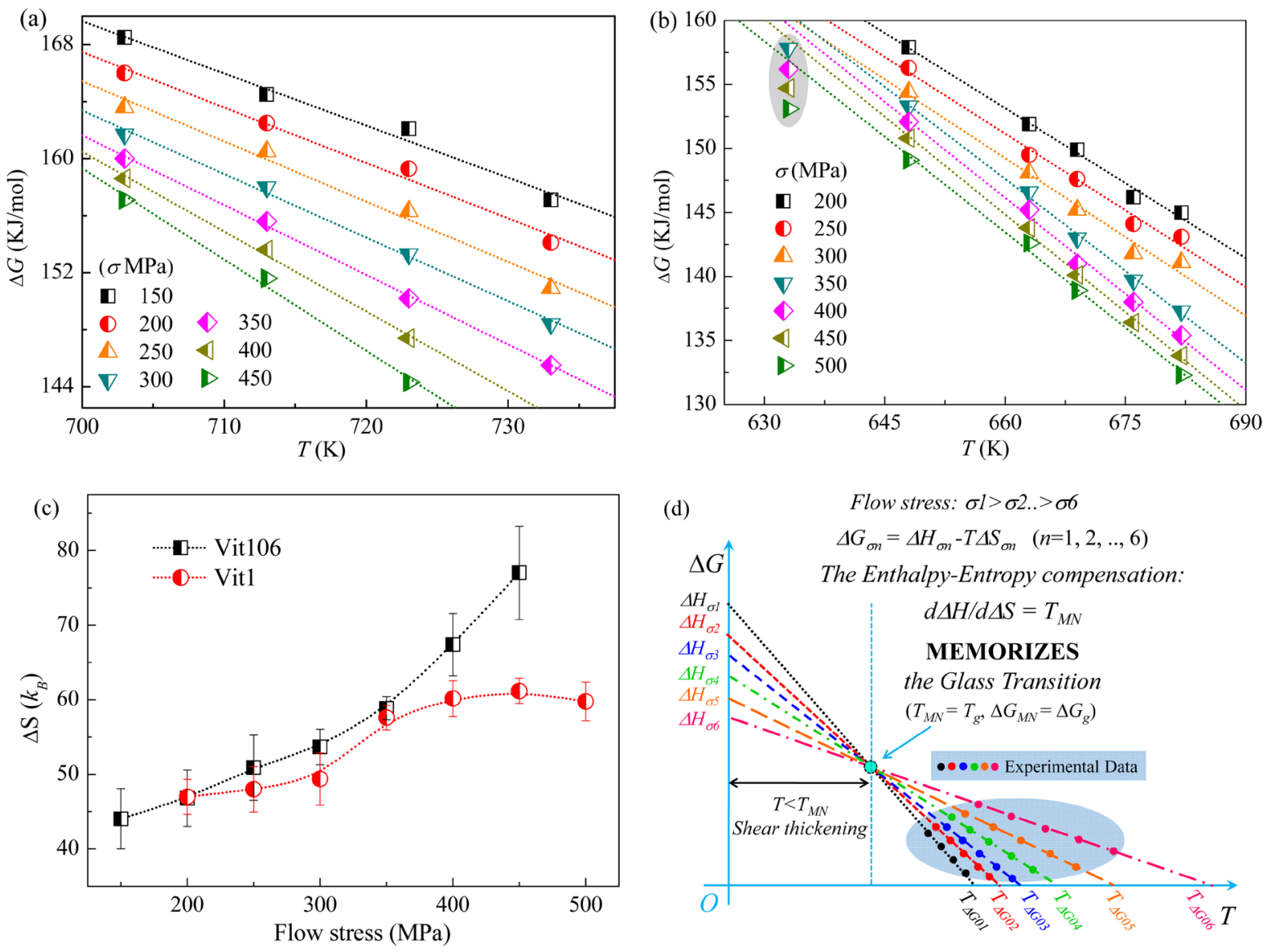

Figure 3. The activation Gibbs free energy versus temperature ( $\Delta G$ versus $T$ ) relations in the shear thinning of Vit106a and Vit1 SMLs: (a) Vit106a, (b) Vit1. The dotted lines are linear fits. The activation entropy $\Delta S$ of Vit106a and Vit1 SMLs at different flow stresses (c), indicating the increased disorder in the shear thinning of SMLs. The dotted lines are eye guides. (d) Illustration of the enthalpy-entropy compensation memorized glass transition.

into a higher activation Gibbs free energy $\Delta G$, for instance $\Delta G_{\sigma 1}>\Delta G_{\sigma 6}$. In the TST, a higher activation Gibbs free energy $\Delta G$ means a lower flow rate and in turn indicates a higher viscosity. This phenomenon is the well known 'shear thickening', where higher flow rate causes higher viscosity. Thus, the enthalpy-entropy compensation law predicts a shear thickening regime in the flow of SMLs on the $\Delta G$ versus $T$ diagram where $T<T_{\mathrm{MN}}$. This shear thickening regime at $T<T_{\mathrm{MN}}$ also supports the above conclusion that the critical point $\left(T_{\mathrm{MN}}, \Delta G_{\mathrm{MN}}\right)$ on the $\Delta G$ versus $T$ diagram is a virtually a kinetic glass transition. To our best knowledge, the shear thickening regime has not been observed in metallic glasses [40]. The reason for the not observed shear thickening regime in metallic glasses is probably due to the fact the homogenous steady state flow of SMLs in the $T<T_{\mathrm{MN}}$ regime (i.e. in glassy state) cannot be reached in real experimental tests, where the highly inhomogeneous shear bands-mediated plastic deformation of metallic glasses [40] dominates the flow. However, the shear thickening phenomenon has already been observed in quite a few glass-like systems [41, 42]. In future, the potential existence of a shear thickening regime in the flow of SMLs is of fundamental significance and worthy of more intensive investigations.

\subsection{Origin of the enthalpy-entropy compensation in SMLS}

Inspired by the microscopic explanation of the enthalpyentropy compensation provided by Yelon and Movaghar [17], the origin of the enthalpy-entropy compensation law in the shear thinning of SMLs can be revealed as follows. As proposed by Boisvert et al [18], the enthalpy-entropy compensation effect in the thermally assisted activation process exhibits a many-body nature, which means that the activation energy for the activation process is provided by multiple typical elementary excitations. To provide the activation energy for the activation process, the multiple excitations are assembled in a collective mode [17]. In the SMLs, the activation process is the flow of SMLs and the typical excitation is the STZ event. In the flow of SMLs, the STZs are assembled in a collective mode [43]. With an increased number of the collectively activated STZs, the number of ways of assembling these STZs (i.e. the routes to the activated state) increases exponentially. It is the growth in the activation routes that results into an increased activation entropy $\Delta S$ under increasing flow stresses which comprises the entropic compensation effect [18].

This proposition has been verified in a model glass where the entropy of combining multiple STZs to overcome the 

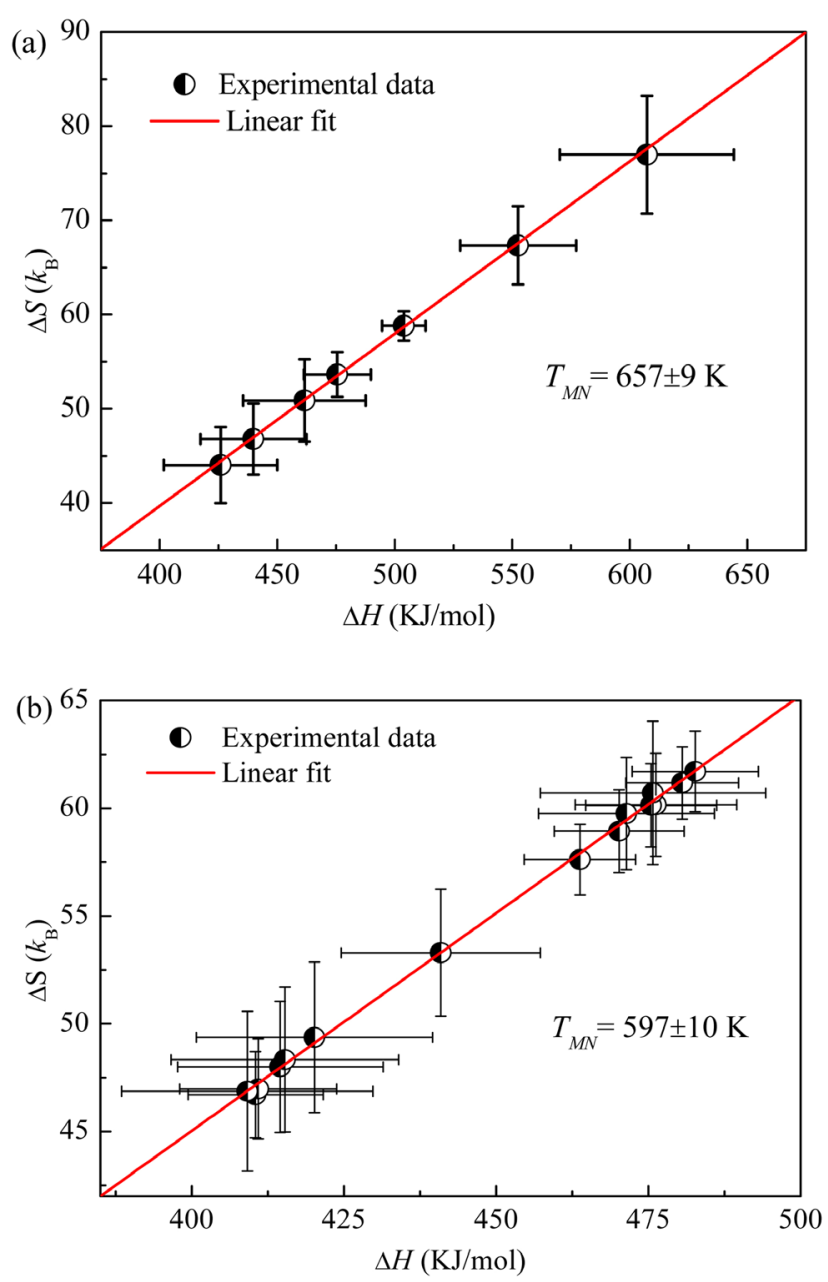

Figure 4. The linear relationship between activation enthalpy $\Delta H$ and activation entropy $\Delta S$ in the shear thinning of Vit106a (a) and Vit1 (b) SMLs. The solid lines are linear fits.

energy barrier for flow constituents the enthalpy-entropy compensation effect [22]. To further confirm this proposition in our work, it is noted that the increased number of collectively activated STZs corresponds to the increment of the activation volume $V$ [17]. The STZs activated in a multipleSTZs collective mode will feel a larger activation volume. Similar collective mode indicated by the increased activation volume is also observed in the nucleation of dislocations [44]. Therefore, the increased $\Delta S$ in the enthalpy-entropy compensation law under increasing flow stress suggests a concomitantly increased activation volume $V$. The activation volume $V=-\partial \Delta G(\sigma, T) / \partial \sigma$ for the flow of the 2 SMLs can be derived via introducing $\Delta G(T, \sigma)=k_{\mathrm{B}} T \ln \left(\dot{\varepsilon}_{0} / \dot{\varepsilon}\right)$. As having been established [45], the activation volume $V$ reads: $V \propto \frac{k_{\mathrm{B}} T}{\sigma m^{*}}$, where $m^{*}=\partial \ln \sigma / \partial \ln \dot{\varepsilon}$ is the strain rate sensitivity index.

For convenience, figure 5 shows the value of $\sigma \cdot m^{*}$ instead of the $k_{\mathrm{B}} T / \sigma m^{*}$ of Vit106a and Vit1 SMLs in steady state flow, respectively, where the main results remain unchanged. It can be seen that for both of the 2 SMLs, $\sigma \cdot m^{*}$ increases, i.e. $V$ decreases, in an initial shear thinning regime $\left(m^{*}>0.4\right.$, as indicated by the dashed line in figures $1(\mathrm{c})$ and (d)). While in a prominent shear thinning regime $m^{*}<0.4, \sigma \cdot m^{*}$

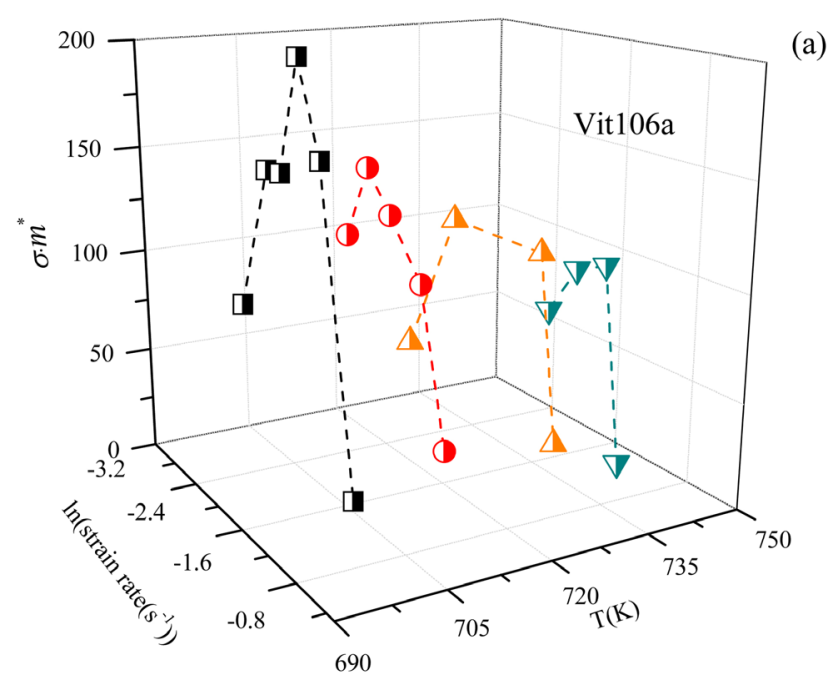

(a)

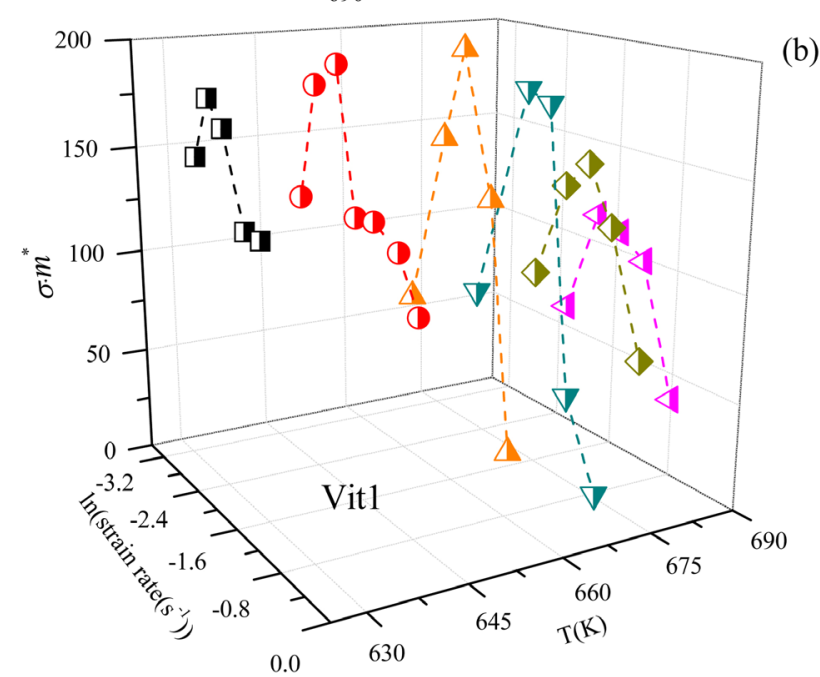

Figure 5. The product of flow stress and strain rate sensitivity index: $\sigma \cdot m^{*}$ at different temperatures of SMLs: (a) Vit106a and (b) Vit1. The dashed lines are eye guides.

decreases, i.e. $V$ increases. The 2 shear thinning regimes have also been observed in the creep of metallic glasses [46], which correspond to 2 different deformation mechanisms, i.e. single atom diffusion (or sparse STZs, $n=1 / m^{*}<2.5$ ) and cluster cooperative shear transformation (or multiple STZs, $n=1 / m^{*}>2.5$ ), respectively. This phenomenon also coincides with the fact that $m^{*} \approx 0.4-0.6$ has been reported as the boundary for the development of long range correlation in the steady flow state of sheared glasses and emulsions $[12,47]$. Thus, the decrease of $\sigma \cdot m^{*}$, i.e. increase of $V$, in the prominent shear thinning regime $m^{*}<0.4$ indicates the development of strong correlation effects between STZs [48], which would lead to the collective activation of STZs. The larger $V$ in the collective activation of STZs, the more routes to the activated state. Hence, the increment of $V$ is concluded to be the main cause for the increment of $\Delta S$ at higher flow stresses in the prominent shear thinning regime $m^{*}<0.4$ of SMLs, i.e. the origin of the enthalpy-entropy compensation. Additionally, it is intriguing to see the initial shear thinning regime $m^{*}>0.4$, where $\sigma \cdot m^{*}$ increases, i.e. $V$ decreases. In this regime, as predicted in simulations and emulsions [12,47], the correlation effect is not dominant. The enthalpy-entropy 
compensation effect, i.e. the increment of $\Delta S$ at increasing flow stresses, is probably due to the increased number of the sparsely distributed STZs arising from the facilitation effect in the activation STZs. The result in figure 5 is in alignment with the competition between the facilitation and correlation effects in the flow of SMLs [48]. These findings enrich current understanding on the many-body nature of the ubiquitous enthalpy-entropy compensation law.

Moreover, to further understand the origin of the enthalpyentropy compensation in SMLs, it is important to note that the compensation effect has also been found to apply to the grain boundary internal friction [49] and dislocation nucleation from the inside of grain boundaries [44]. The atomic structure inside grain boundaries bears a strong resemblance to the amorphous structure of metallic glasses. More importantly, the enthalpy-entropy compensation temperature of the grain boundary internal friction in high purity aluminum also locates at a critical phase transition point [49], below which the relaxation process in grain boundary internal friction with a lower activation enthalpy is faster, while above which the relaxation process with a higher activation enthalpy is faster. This crossover of the relaxation time of the relaxation process in grain boundary internal friction at the compensation temperature is much similar to the memorized glass transition in the current work, below which the flow of SMLs with a lower activation enthalpy is faster, while above which the flow of SMLs with a higher activation enthalpy is faster. The synchronization (i.e. a collective mode) of the relaxation units in the grain boundary internal friction is also considered crucial in the crossover of the relaxation time [49]. On the other hand, the nucleation mechanism of dislocations from the inside of grain boundaries also exhibits a transition from a shufflingassisted mode to a collective nucleation mode upon increasing flow rate [44]. This fact highly coincides with the transition from sparsely activated STZs to the collectively activated STZs in the flow mechanism of SMLs. Especially, similar variation tendency of the activation volume shown in figure 5 is also found for the dislocation nucleation from the inside of grain boundaries. More works on these issues in future would largely advance our knowledge on the universality of the enthalpy-entropy compensation law.

\section{Conclusion}

In summary, the enthalpy-entropy compensation law are examined in the shear thinning regime of 2 typical SMLs. A group of $\Delta G$ versus $T$ relations diverging from each other towards higher temperature, i.e. converging towards lower temperature, are observed in the shear thinning of SMLs. The $\Delta G$ versus $T$ relations indicate an increasing activation entropy $\Delta S$, i.e. increasing disorder, under increasing flow stress and suggest a polyamorphic transition in the yield of metallic glasses. The enthalpy-entropy compensation memorizes the glass transition in the strongly shear-thinned SMLs, demonstrating a kinetic nature of glass transition. The enthalpyentropy compensation effect is attributed to the facilitation effect and the correlation effect in the activation of STZs, in the initial shear thinning regime and in the prominent shear thinning regime divided by a critical strain rate sensitivity index $m^{*} \approx 0.4$, respectively.

\section{Acknowledgments}

This work was financially supported by the National Nature and Science Foundation of China under grant No. 51701082 and by 'the Fundamental Research Funds for the Central Universities'.

\section{ORCID iDs}

Meng Zhang (1) https://orcid.org/0000-0002-2439-5452

\section{References}

[1] Berthier L and Biroli G 2011 Theoretical perspective on the glass transition and amorphous materials Rev. Mod. Phys. 83 587-645

[2] Debenedetti P G and Stillinger F H 2001 Supercooled liquids and the glass transition Nature 410 259-67

[3] Angell C A, Ngai K L, McKenna G B, McMillan P F and Martin S W 2000 Relaxation in glassforming liquids and amorphous solids J. Appl. Phys. 88 3113-57

[4] Yamamoto R and Onuki A 2000 Dynamics of highly supercooled liquids far from equilibrium J. Phys.: Condens. Matter 12 6323-34

[5] Berthier L and Barrat J-L 2002 Shearing a glassy material: numerical tests of nonequilibrium mode-coupling approaches and experimental proposals Phys. Rev. Lett. 89095702

[6] Fuchs M and Cates M E 2002 Theory of nonlinear rheology and yielding of dense colloidal suspensions Phys. Rev. Lett. 89248304

[7] O'Hern C S, Liu A J and Nagel S R 2004 Effective temperatures in driven systems: static versus timedependent relations Phys. Rev. Lett. 93165702

[8] Katgert G, Mobius M E and van Hecke M 2008 Rate dependence and role of disorder in linearly sheared twodimensional foams Phys. Rev. Lett. 101058301

[9] Furukawa A, Kim K, Saito S and Tanaka H 2009 Anisotropic cooperative structural rearrangements in sheared supercooled liquids Phys. Rev. Lett. 102016001

[10] Langer J S 2014 Theories of glass formation and the glass transition Rep. Prog. Phys. 77042501

[11] Viasnoff V and Lequeux F 2002 Rejuvenation and overaging in a colloidal glass under shear Phys. Rev. Lett. 89065701

[12] Haxton T K and Liu A J 2007 Activated dynamics and effective temperature in a steady state sheared glass Phys. Rev. Lett. 99195701

[13] Yu H B, Richert R, Maass R and Samwer K 2015 Unified criterion for temperature-induced and strain-driven glass transitions in metallic glass Phys. Rev. Lett. 115135701

[14] Guan P F, Chen M W and Egami T 2010 Stress-temperature scaling for steady-state flow in metallic glasses Phys. Rev. Lett. 104205701

[15] Liu Y H, Liu C T, Wang W H, Inoue A, Sakurai T and Chen M W 2009 Thermodynamic origins of shear band formation and the universal scaling law of metallic glass strength Phys. Rev. Lett. 103065504

[16] Zhang M, Wang Y M, Li F X, Jiang S Q, Li M Z and Liu L 2017 Mechanical relaxation-to-rejuvenation transition in a Zr-based bulk metallic glass Sci. Rep. 7625 
[17] Yelon A and Movaghar B 1990 Microscopic explanation of the compensation (Meyer-Neldel) rule Phys. Rev. Lett. 65 618-20

[18] Boisvert G, Lewis L J and Yelon A 1995 Many-body nature of the Meyer-Neldel compensation law for diffusion Phys. Rev. Lett. 75 469-72

[19] Ryu S, Kang K and Cai W 2011 Entropic effect on the rate of dislocation nucleation Proc. Natl Acad. Sci. USA 108 5174-8

[20] Gehrig J C, Penedo M, Parschau M, Schwenk J, Marioni M A, Hudson E W and Hug H J 2017 Surface single-molecule dynamics controlled by entropy at low temperatures Nat. Commun. 814404

[21] Yelon A, Movaghar B and Crandall R S 2006 Multi-excitation entropy: its role in thermodynamics and kinetics Rep. Prog. Phys. 69 1145-94

[22] Koziatek P, Barrat J-L, Derlet P and Rodney D 2013 Inverse Meyer-Neldel behavior for activated processes in model glasses Phys. Rev. B 87224105

[23] Falk M L and Langer J S 2011 Deformation and failure of amorphous, solidlike materials Annual Rev. Condens. Matter Phys. 2 353-73

[24] Langer J S 2004 Dynamics of shear-transformation zones in amorphous plasticity: formulation in terms of an effective disorder temperature Phys. Rev. E 70041502

[25] Hedges L O, Jack R L, Garrahan J P and Chandler D 2009 Dynamic order-disorder in atomistic models of structural glass formers Science 323 1309-13

[26] Cheng Y Q and Ma E 2011 Atomic-level structure and structure-property relationship in metallic glasses Prog. Mater. Sci. 56 379-473

[27] Wang Y J, Zhang M, Liu L, Ogata S and Dai L H 2015 Universal enthalpy-entropy compensation rule for the deformation of metallic glasses Phys. Rev. B 92174118

[28] Argon A S and Kuo H Y 1979 Plastic flow in a disordered bubble raft (an analog of a metallic glass) Mater. Sci. Eng. 39 101-9

[29] Argon A S 1979 Plastic deformation in metallic glasses Acta Metall. 27 47-58

[30] Berthier L and Barrat J L 2002 Nonequilibrium dynamics and fluctuation-dissipation relation in a sheared fluid J. Chem. Phys. 116 6228-42

[31] de Hey P, Sietsma J and van den Beukel A 1998 Structural disordering in amorphous $\mathrm{Pd}_{40} \mathrm{Ni}_{40} \mathrm{P}_{20}$ induced by high temperature deformation Acta Mater. 46 5873-82

[32] Tong Y, Iwashita T, Dmowski W, Bei H, Yokoyama Y and Egami T 2015 Structural rejuvenation in bulk metallic glasses Acta Mater. 86 240-6

[33] Lacks D J 2001 Energy landscapes and the non-Newtonian viscosity of liquids and glasses Phys. Rev. Lett. 87225502
[34] Lubchenko V 2009 Shear thinning in deeply supercooled melts Proc. Natl Acad. Sci. USA 106 11506-10

[35] Boots H M J and De Bokx P K 1989 Theory of enthalpyentropy compensation J. Phys. Chem. 93 8240-3

[36] Gallino I, Shah M B and Busch R 2007 Enthalpy relaxation and its relation to the thermodynamics and crystallization of the $\mathrm{Zr}_{58.5} \mathrm{Cu}_{15.6} \mathrm{Ni}_{12.8} \mathrm{Al}_{10.3} \mathrm{Nb}_{2.8}$ bulk metallic glass-forming alloy Acta Mater. 55 1367-76

[37] Busch R, Kim Y J and Johnson W L 1995 Thermodynamics and kinetics of the undercooled liquid and the glass transition of the $\mathrm{Zr}_{41.2} \mathrm{Ti}_{13.8} \mathrm{Cu}_{12.5} \mathrm{Ni}_{10.0} \mathrm{Be}_{22.5}$ alloy J. Appl. Phys. 77 4039-43

[38] McKenna G B 2003 Mechanical rejuvenation in polymer glasses: fact or fallacy? J. Phys.: Condens. Matter 15 S737-63

[39] Evenson Z, Gallino I and Busch R 2010 The effect of cooling rates on the apparent fragility of Zr-based bulk metallic glasses J. Appl. Phys. 107123529

[40] Schuh C A, Hufnagel T and Ramamurty U 2007 Mechanical behavior of amorphous alloys Acta Mater. 55 4067-109

[41] Cheng X, McCoy J H, Israelachvili J N and Cohen I 2011 Imaging the microscopic structure of shear thinning and thickening colloidal suspensions Science 333 1276-9

[42] Fall A, Lemaitre A, Bertrand F, Bonn D and Ovarlez G 2010 Shear thickening and migration in granular suspensions Phys. Rev. Lett. 105268303

[43] Homer E R, Rodney D and Schuh C A 2010 Kinetic Monte Carlo study of activated states and correlated sheartransformation-zone activity during the deformation of an amorphous metal Phys. Rev. B 81064204

[44] Du J-P, Wang Y-J, Lo Y-C, Wan L and Ogata S 2016 Mechanism transition and strong temperature dependence of dislocation nucleation from grain boundaries: an accelerated molecular dynamics study Phys. Rev. B 94104110

[45] Asaro R J and Suresh S 2005 Mechanistic models for the activation volume and rate sensitivity in metals with nanocrystalline grains and nano-scale twins Acta Mater. 53 3369-82

[46] Cao P, Short M P and Yip S 2017 Understanding the mechanisms of amorphous creep through molecular simulation Proc. Natl Acad. Sci. 114 13631-6

[47] Mason T G, Bibette J and Weitz D A 1996 Yielding and flow of monodisperse emulsions J. Colloid Interface Sci. 179 439-48

[48] Zhang M, Liu L and Wu Y 2013 Facilitation and correlation of flow in metallic supercooled liquid J. Chem. Phys. 139164508

[49] Jiang W B, Kong Q P, Molodov D A and Gottstein G 2009 Compensation effect in grain boundary internal friction Acta Mater. 57 3327-31 